\title{
Long-Term Survival (27.7 Years) Following IV Antineoplaston Therapy (ANP) in a 36-Year-Old Female with a Progressive Diffuse Intrinsic Pontine Glioma (DIPG)
}

\author{
Stanislaw R Burzynski, MD, PhD ${ }^{1^{*}}$, Tomasz Janicki ${ }^{1}$, Gregory S Burzynski ${ }^{1}$ and Samuel Beenken ${ }^{2}$
}

${ }^{1}$ Burzynski Clinic, USA

${ }^{2}$ Calera, $A L, U S A$

*Corresponding author: Stanislaw R Burzynski, MD, PhD, Burzynski Clinic, 9432 Katy Freeway, Houston, TX 77055, USA, Tel: 713-335-5697, Fax: 713-335-5658

\begin{abstract}
Background: Long-term survival in Diffuse Intrinsic Pontine Glioma (DIPG) is very rare. The purpose of this report is to detail the early (1988) use of IV Antineoplaston therapy (ANP $\{$ A-10 + AS2-1\}) and its efficacy in DIPG while presenting the 27.7-year survival of a 36-year-old patient treated with ANP for progressive DIPG.

Methods: The patient presented in this manuscript was enrolled in BT-3, a Phase II protocol utilizing IV Antineoplastons $A 10$ and AS2-1 (ANP). Tumor response was measured by bi-monthly MRIs of the brain. All surviving patients were followed for more than three years.

Results: This patient presented to the Burzynski Clinic (BC) with an anaplastic (high-grade) astrocytoma of the pons (DIPG) with resultant paralysis of the right side of the face, diplopia, decrease in strength of the left upper extremity and decreased sensation involving the left upper extremity and left thigh. The patient was experiencing poor balance with difficulty walking, and had dysphagia, right ear hearing loss, memory loss, and headaches. Following ANP, she developed a persistent Complete Response (CR). In BT-3, ANP was administered to a total of 20 patients diagnosed with astrocytomas. Seventeen patients were classified as high grade while three patients were classified as low grade. Four patients achieved a CR, two patients achieved a Partial Response (PR), ten patients had stable disease (SD), and four patients developed progressive disease (PD). Subsequently, one patient having SD achieved a PR. Two patients with SD in BT-3 were subsequently enrolled in a Phase II clinical trial that utilized continuous infusion of higher dose ANP. Both of these patients then developed a CR.
\end{abstract}

Conclusion: BT-3 led to further Phase II clinical studies, which involved a more aggressive use of ANP, including continuous infusions of higher dose A10 and AS2-1 utilizing ambulatory infusion pumps. In addition, there was an increased utilization of ANP in a variety of low- and highgrade brain tumors under the Burzynski Research Institute's (BRl's) IND \# 43,742.

\section{Keywords}

Brain tumor, Anaplastic astrocytoma, Brain stem glioma, Diffuse intrinsic brainstem glioma, Diffuse midline glioma, H3-K27 mutant (DMG H3-K27M), Antineoplaston therapy, Phase II and III clinical studies
Abbreviations
A-10: Antineoplaston A10 (Atengenal); AE: Adverse Event; ANP: IV Antineoplaston Therapy (A-10 + AS2-1); AS2-1: Antineoplaston AS2-1 (Astugenal); Astugenal: Antineoplas- ton AS2-1 (AS2-1); Atengenal: Antineoplaston A10 (A10); BC: Burzynski Clinic; BRI: Burzynski Research Institute; CE: Compassionate Exemption; CNS: Central Nervous System; CR: Complete Response; CTEP: Cancer Therapy Evaluation Program; DIPG: Diffuse Intrinsic Pontine Glio- ma; DMG H3-K27M: Diffuse Midline Glioma, H3-K27 Mu- tant; FDA: Food and Drug Administration; IV: Intravenous; KPS: Karnofsky Performance Status; MRI: Magnetic Res- onance Imaging (of the brain); NIH: National Institutes of Health; OR: Objective Response; OS: Overall Survival; PD: Progressive Disease; PFS: Progression-Free Survival; PR: Partial Response; RT: Radiation Therapy; SD: Stable Dis- ease; TMZ: Temozolomide; WHO: World Health Organiza- tion; UCSF: University of California at San Francisco; VP: Ventriculo-Peritoneal 


\section{Introduction}

Diffuse intrinsic pontine glioma (DIPG) originates in the glial cells of the pons, an integral part of the brain stem. Gliomas can be low- or high-grade based on histological criteria and/or MRI findings. Individuals suffering from DIPG, a high-grade tumor, face a dismal prognosis with a median overall survival (OS) of approximately 11 months, and a 2 year survival rate of $10 \%[1,2]$. Due to the location of the tumor, surgical resection is not possible. To date, radiation therapy (RT) remains the standard of care for newly diagnosed DIPG, but only offers a survival benefit of approximately 3 months [3]. Cytotoxic chemotherapy has not been shown to be effective [2]. There is no standard of care for progressive DIPG after RT.

Phase I clinical studies of Antineoplaston A10 (Atengenal, A-10) and Antineoplaston AS2-1 (Astugenal, AS2-1) demonstrated objective responses (ORs) in the treatment of primary malignant brain tumors, which prompted the selection of these drugs for Phase II clinical studies $[4,5]$.

In 1988, Dr. SR Burzynski developed a Phase II study protocol designated BT-3 and titled "Therapy of Primary Brain Tumors with Antineoplaston A10 and Antineoplaston AS2-1" The objectives of this study were to determine: i) The effectiveness of IV Antineoplaston therapy (ANP $\{A-10+A S 2-1\}$ ) in the control of primary brain tumors and; ii) The toxicity of ANP. The entire BT-3 data set was subsequently published in the Anticancer Section of the Proceedings of the $17^{\text {th }}$ International Congress of Chemotherapy [6].

BT-3 led to further Phase II clinical studies, which involved the more aggressive use of ANP, especially continuous infusions of higher dose A10 and AS2-1, utilizing ambulatory infusion pumps, in accordance with BT-4 [7]. In addition, there was an increased utilization of ANP in a variety of low- and high-grade brain tumors under the Burzynski Research Institute's (BRI's) IND \# 43,742 . Multiple Phase II protocols have now been completed and the impact of ANP on the treatment of brain tumors has been widely published [8-40].

On October 4, 1991, three members of the NIH Cancer Therapy Evaluation Program (CTEP), with an invited neuropathologist and an invited neuroradiologist, visited Dr. Burzynski at the Burzynski Clinic (BC) to review selected brain tumors cases from Phase I and Phase II studies. Following thorough review of seven cases selected by CTEP, five definite or "possible" cases of CR were identified, including the case of a 36-year-old female with DIPG, presented here, who achieved a CR with long term (27.7 years) survival after receiving ANP in BT-3 [41].

\section{Methods}

The patient presented here met the following cri- teria for enrollment in BT-3: i) A measurable low- or high- grade astrocytoma (a glial tumor with astrocytic differentiation); ii) Ineligibility for or refusal of RT, and/ or demonstrated progressive disease (PD) following RT or chemotherapy; iii) A life expectancy of at least three months; iv) A KPS of at least 60; v) A signed informed consent document indicating that she was aware of the investigational nature of ANP, the unpredictable nature of her response to ANP, and the possibility of worsening disease while receiving ANP.

Before ANP began, a subclavian Broviac catheter was placed. Patients $>18$ years of age, received ANP at a starting dose of $0.1 \mathrm{~g} \mathrm{~A}-10$ given as $1 \mathrm{ml}$ IV. If the patient did not show side effects 30 minutes following this injection, the patient received an additional $10.0 \mathrm{~g} \mathrm{~A}-10$ via IV infusion (100 $\mathrm{mg} \mathrm{A-10/ml)} \mathrm{at} 50 \mathrm{ml} / \mathrm{hr}$. In the absence of limiting toxicity, A-10 dosage was subsequently increased by $0.3 \mathrm{~g} / \mathrm{kg} / \mathrm{d}$ until a maximum dosage of $1 \mathrm{~g} /$ $\mathrm{kg} / \mathrm{d}$ was reached. During this escalation, the IV infusion rate was increased to $100 \mathrm{ml} / \mathrm{hr}$.

Once a patient reached his/her maximum dosage of A-10, AS2-1 was added to the therapeutic regime. The starting dose was $0.1 \mathrm{~g}$ AS2-1 given as $1 \mathrm{ml}$ IV. If the patient did not show side effects 30 minutes following this injection, the patient received an additional $10.0 \mathrm{~g}$ AS2-1 via IV infusion (100 mg AS2-1/ml) at $100 \mathrm{ml} / \mathrm{hr}$. In the absence of limiting toxicity, AS2-1 dosage was increased by $0.15 \mathrm{~g} / \mathrm{kg} /$ day until a dosage of $0.4 \mathrm{~g} / \mathrm{kg} /$ day was reached. During this escalation, the IV infusion rate was maintained at $100 \mathrm{ml} / \mathrm{hr}$. A minimum of six months of treatment was necessary for a patient to be evaluable. Patients were removed from treatment secondary to progressive disease, unacceptable toxicity, or patient request.

Tumor response to ANP was measured utilizing MRIs of the brain performed every two months. The MRIs performed at the start of BT-3 were without gadolinium contrast as it was not yet in routine use. The response criteria were as follows: A complete response (CR) indicated complete disappearance of all tumor while a partial response $(P R)$ indicated $a \geq 50 \%$ reduction in tumor size as determined by the product of its longest perpendicular diameters. PD indicated $a \geq 50 \%$ increase in tumor size as determined by the product of its longest perpendicular diameters while stable disease (SD) did not meet the criteria for PR or PD. Tumor response to ANP was measured independently by a local neuroradiologist and by three outside neuroradiologists.

\section{Results}

At the time of her presentation at $B C$, the patient was 36 years of age. She had been in good health until just prior to July 21, 1987 when she was diagnosed by MRI and stereotactic biopsy with an anaplastic (high grade) astrocytoma of the pons (DIPG). Genomic studies, such as mutation of $\mathrm{H} 3 \mathrm{~K} 27$, were not performed at the time 


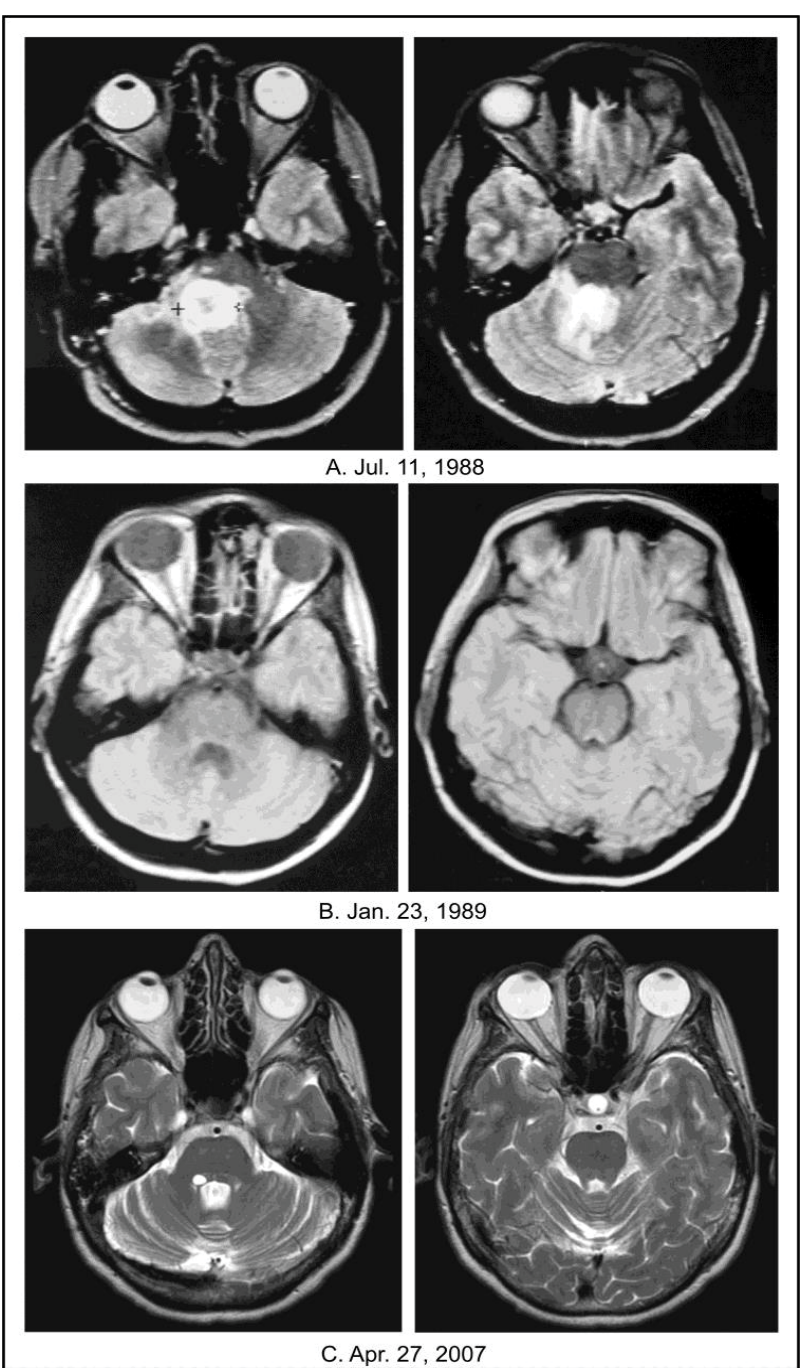

Figure 1: Achievement of a $C R$ in an adult female patient with progressive DIPG. A) The baseline images dated July 11,1988 show a right-sided pontine tumor measuring 3.4 $\mathrm{cm} \times 2.1 \mathrm{~cm}\left(7.14 \mathrm{~cm}^{2}\right)$; B) The images dated January 23, 1989 show achievement of a CR after 6 months of ANP on protocol BT-3; C) The images dated April 27, 2007 show long-term persistence of the CR. All images are T2weighted and FLAIR.

CR: Complete Response; DIPG: Diffuse Intrinsic Brainstem Glioma; ANP: Antineoplaston Therapy (A-10 + AS2-1).

of biopsy because such technology was not available. Recently, genomic analysis of the archival specimen was attempted, but was unsuccessful due to an insufficient amount of preserved tissue. From August 31, 1987 until October 23, 1987, the patient was treated with 78 cGy of hyperfractionated RT at UCSF. She did not receive further treatment at that time despite evidence of PD documented by MRIs of the brain on February 5, 1988 and April 29, 1988.

Physical examination at $\mathrm{BC}$ revealed paralysis of the right side of the face, diplopia, decrease in strength of the left upper extremity and decreased sensation involving the left upper extremity and left thigh. She was experiencing poor balance with difficulty walking, and had dysphagia, right ear hearing loss, memory loss, and headaches. Her KPS was 60.
The MRI of the brain performed on April 29, 1988, which confirmed PD after hyperfractionated RT, resulted in off-protocol treatment at BC with A10, AS2-1, and low dose oral methotrexate. MRI of the brain performed on July 11, 1988 (see Figure 1) showed a right brainstem lesion measuring $3.4 \mathrm{~cm} \times 2.1 \mathrm{~cm}\left(7.14 \mathrm{~cm}^{2}\right)$ and the patient was enrolled in BT-3. IV dexamethasone was stopped on September 9, 1988 with the patient receiving no steroids after that date.

On December 1, 1988, after $\sim 4$ months of protocol treatment on BT-3, the patient achieved a PR ( $58 \%$ decrease in two-dimensional size), as demonstrated by non-contrast MRI of the brain. On January 23, 1989, after $\sim$ six months of protocol treatment, the patient achieved a CR, as demonstrated by non-contrast MRI of the brain (see Figure 1). Beginning in 1989, the CR was also demonstrated by multiple gadolinium-enhanced MRIs. ANP infusions were permanently discontinued on August 10, 1989 and were followed by maintenance oral A10 and AS2-1, which were discontinued on January 21,1990 . As discussed previously, in October 1991, the MRI findings of a CR were reviewed and confirmed by CTEP [41].

The following AEs, possibly attributable to ANP, occurred while the patient was treated on BT-3: Mild leukopenia, headaches, dizziness, skin rash, and fever.

On March 3, 2016, the patient passed away after acquiring influenza and developing a pneumonia. Her death was not related to her previous brain tumor. She had lived a good life, of almost 30 years, after her brain tumor diagnosis.

In BT-3, ANP was administered to 20 patients diagnosed with astrocytomas. Seventeen patients were classified as high grade while three patients were classified as low grade. Four patients achieved a CR, two patients achieved a PR, ten patients had SD, and four patients developed PD [6]. Subsequently, one patient having SD achieved a PR. All surviving patients were followed for more than three years. Two patients with SD in BT-3 were subsequently enrolled in a Phase II clinical trial that utilized continuous infusion of higher dose ANP. Both of these patients then developed a CR $[7,8]$.

\section{Discussion}

Brainstem gliomas represent 10 to $20 \%$ of primary tumors of the central nervous system and are diagnosed primarily in children with a median age of onset of 6 to 7 years [42]. The number of children in the USA with brainstem gliomas is approximately $660[42,43]$ and most occur in the pons. Brainstem gliomas affect the cranial nerves, causing symptoms such as diplopia, the inability to fully close the eyelids, drooping of one side of the face, dysphagia, and difficulty chewing. These tumors also affect the "long tracks" of the brain, with resultant weakness of the arms and/or legs. 
At the time of initial diagnosis, these tumors are usually confined to the brainstem. When the tumor progresses, the progression is usually contiguous. Metastasis via the subarachnoid space has been reported in up to $30 \%$ of cases and usually occurs at the same time as local progression. There is no generally applied staging system for childhood brainstem gliomas.

Due to its anatomical location, diagnostic biopsies of pontine gliomas are difficult to obtain. Diagnosis has frequently been based on MRI alone. However, routine biopsy of children with suspected DIPG has been performed in Europe since 2003 [44]. In a report detailing their experience in 24 children, morbidity was reported in 2 children (cranial nerve palsy, worsening hemiparesis), which was reversible, and resulted in no mortality. The investigators concluded that the procedure was safe in experienced hands using modern neurosurgical technique [44]. Given this demonstration of safety, there is a push within the United States pediatric neuro-oncology community for routine biopsy of patients with suspected DIPG [45].

MRI identifies four different types of brainstem gliomas: focal, cervicomedullary, dorsal exophytic, and DIPG. With the increased use of biopsy for the diagnosis of DIPG, the World Health Organization (WHO), in 2016, reclassified the majority of DIPG within a novel tumor entity: diffuse midline glioma, H3-K27 mutant (DMG H3-K27M). This entity is defined as an infiltrative highgrade glioma, located in the brain midline, i.e. usually brainstem, spinal cord, cerebellum or thalamus, with astrocytic differentiation and $\mathrm{K} 27 \mathrm{M}$ mutation in either H3F3A or HIST1H3B/C [46]. Up to 85\% of DIPGs harbor this mutation $[47,48]$. Wild-type H3-K27 DIPG has not yet been separately classified within the revised WHO classification, but shows similar survival as mutant H3K27M DIPG [49].

A six-week course of conventional RT is standard treatment for DIPG and frequently will result in improvement of signs and symptoms. Unfortunately, the signs and symptoms usually recur after six to nine months, concomitant with PD. Survival past 14 to 18 months is uncommon. Cytotoxic chemotherapy has not been effective in the treatment of DIPG.

ANP's mechanism of action differs from that of RT or cytotoxic chemotherapy. Growth of normal cells is controlled by cell cycle progression genes (oncogenes) and by cell cycle arrest genes (tumor suppressor genes). In cancer, alteration of these control genes in malignant cells favors aggressive cell proliferation. Evidence suggests that ANP affects 112 genes in the tumor genome and functions as "molecular switches" which "turn on" tumor-suppressor genes and "turn off" oncogenes. Hence, the antineoplastic action of ANP therapy in DIPG involves restoration of cell cycle control, induction of programmed cell death, and interference with cancer cell metabolism and nuclear transport [50].
Adult brainstem glioma constitutes less than $2 \%$ of adult gliomas, with a slight male preponderance. Most cases consist of grade II oligodendrogliomas or astrocytomas. Adult DIPG (patient age $>21$ years) is very uncommon with reports of its yearly incidence in the U.S. ranging from 20-100 cases. The most frequent presenting symptom for adult brainstem glioma is headache. Cranial nerve deficits and long tract signs are also common. "Crossed" deficits, in which facial signs and symptoms are contralateral from arm/leg signs and symptom, can be seen. Occasionally clinical decline can precede radiographic progression, as the proximity of critical structures in the brainstem leaves little room for growth before deficits occur $[51,52]$.

A multicenter, retrospective analysis of all cases of adult brainstem gliomas identified $(n=101)$ was performed [53]. The radiographic appearance of adult brainstem glioma varied, with approximately $40 \%$ of these tumors demonstrating gadolinium enhancement. Brainstem gliomas were centered in the pons in approximately $60 \%$ of adult cases, but also arose from the midbrain or medulla, and infiltrated beyond the brainstem. These could be exophytic or infiltrative and diffuse with little mass effect.

A retrospective analysis of all adult brainstem glioma patients $(n=143)$ at MD Anderson Cancer Center demonstrated a median OS of 21.1 months for WHO grade 3 anaplastic astrocytoma (including DIPG), and 14.8 months for glioblastoma. Increasing age was associated with worsened survival [54].

\section{Conclusion}

In this report, we have presented the case of a 36-year-old female patient, with recurrent Anaplastic (high-grade) astrocytoma of the pons (DIPG), who was treated with ANP on Protocol BT-3 and survived for 27.7 years. BT-3 led to further Phase II clinical studies, which involved a more aggressive use of ANP, including continuous infusions of higher dose A10 and AS2-1 utilizing ambulatory infusion pumps. In addition, there was an increased utilization of ANP in a variety of low- and high-grade brain tumors under BRI's IND \# 43,742. Multiple Phase II protocols have now been completed and the impact of ANP on the treatment of brain tumors has been widely published [8-40].

Based on our prior experience and success utilizing ANP in the treatment of progressive DIPG, we have collaborated with the FDA to develop two additional DIPG protocols. The first, BRI-BT-52, "A Randomized Phase 3 Study of Combination Antineoplaston Therapy [Antineoplastons A10 (Atengenal) and AS2-1 (Astugenal)] Plus Radiation Therapy vs. Radiation Therapy Only in Subjects with Newly Diagnosed Diffuse, Intrinsic, Brainstem Glioma" awaits IRB approval. The second, BRI-BT-55, "A Phase 2 Study of Atengenal (A-10) and Astugenal (AS21) in Diffuse, Intrinsic, Brainstem Glioma (DIPG)" has 
received IRB-approval and will be opened for patient accrual in the near future.

\section{Acknowledgments}

The authors express their appreciation to Carolyn Powers for preparation of the manuscript and to Ramiro Rivera, Mohamed Khan, Jennifer Pineda and Adam Golunski for their involvement.

\section{References}

1. Hoffman LM, Veldhuijzen van Zanten SEM, Colditz N, Baugh J, Chaney B, et al. (2018) Clinical, radiologic, pathologic, and molecular characteristics of long-term survivors of diffuse intrinsic pontine glioma (DIPG): A collaborative report from the International and European Society for Pediatric Oncology DIPG Registries. J Clin Oncol 36: 19631972.

2. Jansen MH, Veldhuijzen van Zanten SE, Aliaga ES, Heymans MW, Warmuth-Metz M, et al. (2015) Survival prediction model of children with diffuse intrinsic pontine glioma based on clinical and radiological criteria. Neuro Oncol 17: 160-166.

3. Langmoen IA, Lundar T, Storm-Mathisen I, Lie SO, Hovind $\mathrm{KH}$ (1991) Management of pediatric pontine gliomas. Childs Nerv Syst 7: 13-15.

4. Burzynski SR, Burzynski B, Mohabbat MO (1986) Toxicology studies on antineoplaston AS2-1 injections in cancer patients. Drugs Exp Clin Res 25-35.

5. Burzynski SR, Kubove E (1986) Toxicology studies on antineoplaston A10 injections in cancer patients. Drugs Exp Clin Res 47-55.

6. Burzynski SR, Kubove E, Burzynski B (1992) Phase II clinical trials of antineoplastons A10 and AS2-1 infusions in astrocytoma. In: Adam D, Recent Advances in Chemotherapy. Munich Futuramed Publishers.

7. Burzynski SR, Kubove E, Szymkowski B (1993) Phase II clinical trials of antineoplaston A10 and AS2-1 infusions in high grade glioma. Presented at the 18th International Congress of Chemotherapy, Stockholm, Sweden.

8. Burzynski SR, Conde AB, Peters A, Saling B, Ellithorpe R, et al. (1999) A retrospective study of antineoplastons A10 and AS2-1 in primary brain tumors. Clin Drug Investig 18: 1-10.

9. Burzynski SR, Weaver RA, Bestak M, Lewy RI, Janicki TJ, et al. (2003) Phase II study of Antineoplastons A10 and AS2-1 (ANP) in children with recurrent and progressive multicentric glioma. A preliminary report. Neuro-Oncology 5: 358.

10. Burzynski SR, Lewy RI, Weaver R, Janicki T, Jurida G, et al. (2004) Long-term survival and complete response of a patient with recurrent diffuse intrinsic brain stem glioblastoma multiforme. Integ Cancer Ther 3: 257-261.

11. Burzynski SR, Weaver R, Lewy R, Janicki T, Jurida G, et al. (2004) Phase II study of antineoplaston A10 and AS2-1 in children with recurrent and progressive multicentric glioma. A preliminary report. Drugs RD 5: 315-326.

12. Burzynski SR, Weaver R, Bestak M, Janicki T, Jurida G, et al. (2004) Phase II studies of antineoplastons A10 and AS2-1 (ANP) in children with atypical teratoid/rhabdoid tumors (AT/RT) of the central nervous system. A preliminary report. Neuro-Oncology 6: 427.

13. Burzynski SR, Weaver R, Bestak M, Janicki T, Szymkowski
B, et al. (2004) Treatment of primitive neuroectodermal tumors (PNET) with antineoplastons A10 and AS2-1 (ANP). Preliminary results of phase II studies. Neuro-Oncology 6: 428.

14. Burzynski SR, Weaver RA, Janicki T, Szymkowski B, Jurida $G$, et al. (2005) Long- term survival of high-risk pediatric patients with primitive neuroectodermal tumors treated with antineoplastons A10 and AS2-1. Integr Cancer Ther 4: 168177.

15. Burzynski SR (2006) Targeted therapy for brain tumors. In: Yang AV, Brain cancer therapy and surgical interventions. New York Nova Science Publishers.

16. Burzynski SR, Janicki TJ, Weaver RA, Burzynski B (2006) Targeted therapy with antineoplastons A10 and AS2-1 of high grade, recurrent, and progressive brainstem glioma. Integr Cancer Ther 5: 40-47.

17. Burzynski SR (2006) Treatments for astrocytic tumors in children: Current and emerging strategies. Paediatr Drugs 8: 167-168.

18. Burzynski SR (2007) Recent clinical trials in diffuse intrinsic brainstem glioma. Cancer Ther 5: 379-390.

19. Burzynski S, Janicki T, Burzynski G, Marszalek A (2013) Long-term survival ( $>13$ years) in a child with recurrent diffuse pontine gliosarcoma: A case report. J Ped Hematol Oncol 36: e433-e439.

20. Burzynski SR, Janicki TJ, Burzynski GS, Marszalek A (2014) A phase II study of antineoplastons A10 and AS21 in children with high-grade glioma. Final report (Protocol BT-06) and review of recent trials. J Cancer Ther 5: 565577.

21. Burzynski SR, Janicki TJ, Burzynski GS (2014) A phase II study of antineoplastons A10 and AS2-1 in adult patients with recurrent glioblastoma multiforme: Final report (Protocol BT-21). J Cancer Ther 5: 946-956.

22. Burzynski SR, Burzynski GS, Janicki TJ (2014) Recurrent glioblastoma multiforme-A strategy for long-term survival. J Cancer Ther 5: 957-976.

23. Burzynski SR, Janicki TJ, Burzynski GS, Marszalek A, Brookman S (2014) A phase II study of antineoplastons A10 and AS2-1 in children with recurrent, refractory or progressive primary brain tumors-Final report (Protocol BT22). J Cancer Ther 5: 977-988.

24. Burzynski SR, Janicki TJ, Burzynski GS, Brookman S (2014) Preliminary findings on the use of targeted therapy with pazopanib and other agents in combination with sodium phenylbutyrate in the treatment of glioblastoma multiforme. J Cancer Ther 5: 1423-1437.

25. Burzynski GS, Janicki TJ, Marszalek A, Burzynski S (2014) Long-term survival ( $>20$ years) of a child with brainstem glioma treated with antineoplastons A10 and AS2-1: A case report. Neuro Oncol 16.

26. Burzynski SR, Janicki TJ, Burzynski GS, Marszalek A (2014) The response and survival of children with recurrent diffuse intrinsic pontine glioma based on phase II study of antineoplastons $A 10$ and AS2-1 in patients with brainstem glioma. Childs Nerv Syst 30: 2051-2061.

27. Burzynski SR, Burzynski G, Janicki J, Marszalek A (2015) Complete response and long-term survival ( $>20$ years) of a child with tectal glioma: A case report. Pediatr Neurosurg 50: $99-103$

28. Burzynski SR, Janicki TJ, Burzynski G (2015) A phase II study of Antineoplastons A10 and AS2-1 injections in adult 
patients with recurrent anaplastic astrocytoma - Final report (Protocol BT-15). Cancer Clin Oncol 4: 13-23.

29. Burzynski SR, Janicki TJ, Burzynski GS, Marszalek A (2015) A phase II study of antineoplastons A10 and AS21 in adult patients with newly-diagnosed anaplastic astrocytoma-final report (Protocol BT-08). Cancer Clin Oncol 4 28-38.

30. Burzynski SR, Burzynski GS, Marszalek A, Janicki J, Martinez-Canca J (2015) Long-term survival (over 20 years), complete response and normal childhood development in medulloblastoma treated with Antineoplastons A10 and AS2-1. J Neurol Stroke 2.

31. Burzynski SR, Burzynski GS, Marszalek A, Janicki TJ, Martinez-Canca JF (2015) Long-term survival over 21 years and pathologically confirmed complete response in pediatric anaplastic astrocytoma: A case report. J Neurol Stroke 2.

32. Burzynski SR, Janicki T, Burzynski G (2015) Comprehensive genomic profiling of recurrent classic glioblastoma in a patient surviving eleven years following antineoplaston therapy. Cancer Clin Oncol 4: 41-52.

33. Burzynski SR, Burzynski GS, Brookman S (2015) A case of sustained objective response of recurrent/progressive diffuse intrinsic pontine glioma with phenylbutyrate and targeted agents. J Cancer Ther 6: 40-44.

34. Burzynski SR, Janicki T, Burzynski G, Marszalek A (2015) A phase II study of antineoplastons A10 and AS2-1 in patients with brainstem gliomas. The report on non-diffuse intrinsic pontine glioma (Protocol BT-11). J Cancer Ther 6: 334-344.

35. Burzynski SR, Janicki T, Burzynski G (2015) A phase II study of antineoplastons A10 and AS2-1 in adult patients with primary brain tumors. Final report (Protocol BT-09). J Cancer Ther 6: 1063-1074.

36. Burzynski SR, Janicki TJ, Burzynski GS (2016) Primary CNS tumors and leptomeningeal, disseminated and/or multicentric disease in children treated in Phase II studies with antineoplastons A10 and AS2-1. Cancer Clin Oncol 5: 38-48.

37. Burzynski SR, Janicki TJ, Burzynski GS (2016) A phase II study of antineoplastons A10 and AS2-1 in children with low-grade astrocytomas-Final report (Protocol BT-13). J Cancer Ther 7: 837-850.

38. Burzynski SR, Janicki TJ, Burzynski GS (2017) Antineoplastons $A 10$ and AS2-1 in the treatment of children with optic pathway glioma: Final Report for protocol BT-23. Cancer Clin Oncol 6: 25-35.

39. Burzynski SR, Janicki TJ, Burzynski GS, Marszalek A (2017) A phase II study of Antineoplastons A10 and AS2-1 in children with brain tumors. Final Report (Protocol BT-10). J Cancer Ther 8: 173-187.

40. Burzynski SR, Janicki T, Beenken, S (2019) Treatment of recurrent glioblastoma multiforme ( $\mathrm{GBBM}$ ) with antineoplaston AS2-1 in combination with targeted therapy. Cancer Clin Oncol 8

41. Hawkins MG, Friedman MA (1992) National Cancer Institute's evaluation of unconventional cancer treatments. $J$ Natl Cancer Inst 84: 1699-1702.

42. Smith MA, Freidlin B, Ries LA, Simon R (1998) Trends in reported incidence of primary malignant brain tumors in children in the United States. J Natl Cancer Inst 90: 12691277.
43. Central Brain Tumor Registry of the United States (CBTRUS) 2005-2006. Primary brain tumors in the United States statistical report, years data collected 1998-2002.

44. Roujeau T, Machado G, Garnett M, Miquel C, Puget S, et al. (2007) Stereotactic biopsy of diffuse pontine lesions in children. J Neurosurg 107: 1-4.

45. Cage TA, Samagh SP, Mueller S, Nicolaides T, Haas-Kogan D, et al. (2013) Feasibility, safety, and indications for surgical biopsy of intrinsic brainstem tumors in children. Childs Nerv Syst 29: 1313-1319.

46. Louis DN, Perry A, Reifenberger G, von Deimling A, Figarella-Branger D, et al. (2016) The 2016 World Health Organization classification of tumors of the central nervous system: A summary. Acta Neuropathol 131: 803-820.

47. Castel D, Philippe C, Calmon R, Le Dret L, Truffaux N, et al. (2015) Histone H3F3A and HIST1H3B K27M mutations define two subgroups of diffuse intrinsic pontine gliomas with different prognosis and phenotypes. Acta Neuropathol 130: 815-827.

48. Karremann M, Gielen GH, Hoffmann M, Wiese M, Colditz $\mathrm{N}$, et al. (2018) Diffuse high-grade gliomas with H3 K27M mutations carry a dismal prognosis independent of tumor location. Neuro-Oncol 20: 123-131.

49. Von Bueren AO, Karremann M, Gielen GH, Benesch M, Fouladi M, et al. (2018) A suggestion to introduce the diagnosis of "diffuse midline glioma of the pons, H3 K27 wildtype (WHO grade IV)". Acta Neuropathol 136: 171-173.

50. Burzynski SR, Patil S (2014) The effect of antineoplastons A10 and AS2-1 and metabolites of sodium phenylbutyrate on gene expression in glioblastoma multiforme. J Cancer Ther 5: 929-945.

51. Salmaggi A, Fariselli L, Milanesi I, Lamperti E, Silvani A, et al. (2008) Natural history and management of brainstem gliomas in adults. A retrospective Italian study. J Neurol 255: 171-177.

52. Reyes-Botero G, Mokhtari K, Martin-Duverneuil N, Delattre JY, Laigle-Donadey F (2012) Adult brainstem gliomas. Oncologist 17: 388-397.

53. Kesari S, Kim RS, Markos V, Jan Drappatz, Patrick Y Wen, et al. (2008) Prognostic factors in adult brainstem gliomas: A multicenter retrospective analysis of 101 cases. J Neuro-oncol 88: 175-183.

54. Theeler BJ, Ellezam B, Melguizo I, John F de Groot, Anita Mahajan, et al. (2015) Adult brainstem gliomas: Correlation of clinical and molecular features. J Neurol Sci 353: 92-97. 